\title{
Primary Peritoneal Serous Carcinoma in Men: $A$ Rare and Non-BRCA-associated Entity
}

\author{
SUSAN L. NEUHAUSEN ${ }^{1}$, HAGIT SHANI ${ }^{2}$, LITAL KEINAN BOKER ${ }^{3}$, LINDA STEELE $^{1}$, \\ BARBARA G. SILVERMAN ${ }^{3}$, LAURA OTTINI ${ }^{4}$, VALENTINA SILVESTRI ${ }^{4}$, \\ YAEL LAITMAN $^{2}$, JACOB KORACH $^{5,6}$, TAMAR PERRI $^{6}$ and EITAN FRIEDMAN ${ }^{2,5,7}$ \\ ${ }^{1}$ Department of Population Sciences, Research Institute, City of Hope, Duarte, CA, U.S.A.; \\ ${ }^{2}$ Oncogenetics Unit, ${ }^{5}$ Meirav High-risk Clinic, ${ }^{6}$ Gyneco-oncology Department, \\ Chaim Sheba Medical Center, Tel-Hashomer, Israel; \\ ${ }^{3}$ Israeli National Cancer Registry, Tel-Hashomer, Israel; \\ ${ }^{4}$ Department of Molecular Medicine, La Sapienza University of Rome, Rome, Italy; \\ ${ }^{7}$ Department of Genetics and Biochemistry, Sackler School of Medicine, Tel-Aviv University, Tel-Aviv, Israel
}

\begin{abstract}
Background: Primary peritoneal serous carcinoma (PPSC) is a rare neoplasm. The paucity of reported cases among men may provide insight to the cell of origin of PPSC. Materials and Methods: A search for the ICD 0-3 code of PPSC (C48.2) in the following datasets: the Israeli National Cancer registry (INCR), the Surveillance, Epidemiology, and End Results (SEER) database in the USA, Israeli male BRCA carriers, male high-risk and BRCA carriers in a USA study, and the Italian Study on Male Breast Cancer (MBC) were performed. Results: In the INCR dataset, 220 entries for C48.2 code were noted, with only one male (male:female ratio $=0.0045)$. In the SEER dataset for histology codes of papillary/serous/ adenocarcinoma, 2,673 cases were recorded, with five males (male:female ratio=0.0018). None of the recorded US or Italian male BRCA carriers or $M B C$, or Israeli male BRCA carriers was diagnosed with PPSC. Conclusion: PPSC is a rare neoplasm, seemingly not associated with BRCA mutations in men, and fallopian tube epithelial cell implants may contribute to its development.
\end{abstract}

Primary peritoneal serous carcinoma (PPSC), or papillary serous carcinoma of the peritoneum, is a rare, intraabdominal neoplasm, with an age-adjusted incidence rate in the US is 6.78 per million (1). PPSC diagnosis carries a

Correspondence to: Eitan Friedman, MD, Ph.D., Head, the Susanne Levy Gertner Oncogenetics Unit, Sheba Medical Center, Tel Hashomer, Israel. Tel: +972 35303173, Fax: +972 35357308, Mobile: +972 522891561, e-mail feitan@post.tau.ac.il or eitan.friedman@sheba.health.gov.il

Key Words: Primary peritoneal serous carcinoma, PPSC, ovarian cancer, male $B R C A$ mutations, cancer risk. grave prognosis, with a 5-year survival of less than $20 \%$ in affected women (2). PPSC has been presumed to arise from extraovarian mesothelium that has a Mullerian potential or endometriosis involving the peritoneal surface (3-5). Yet because of its rarity, little is known about the molecular mechanisms that underlie the development of PPSC.

PPSC is overwhelmingly diagnosed in women, with a propensity in female $B R C A 1$ or $B R C A 2$ gene mutation carriers, even after risk-reducing bilateral salpingooophorectomy (RRBSO). Casey and co-workers reported a $3.5 \%$ risk over 20 years for developing PPSC in BRCA mutation carriers after RRBSO, with more cases noted in $B R C A 1$ carriers compared to BRCA2 carriers (6). Rebbeck et $a l$. reported a rate of $0.8 \%$ in $B R C A$ carriers diagnosed up to 8.6 years after RRBSO (7). Among unselected, average-risk women who underwent salpingo-oophorectomy for noncancer reasons, the rate of PPSC is minimal: $1 / 4128$ cases with a median follow-up of 7.2 years after BSO that was carried out not in the context of risk reduction (8). The paucity of reported cases among men (9-11) raises interesting issues: Should PPSC be included in the spectrum of cancers that affect men as well as women? What is the rate of this rare tumor in male $B R C A$ carriers and males from high-risk, non- $B R C A$ families? What is the gender ratio of this diagnosis on a national cancer registry level? The current study was undertaken to shed light on these issues in order to provide more insight into the pathogenesis of these tumors.

\section{Materials and Methods}

In order to maximize the ability to analyze the rate of PPSC in males,we queried several datasets: the INCR and the Surveillance, Epidemiology, and End Results (SEER) datasets (datasets that include all consecutive cancer diagnoses in Israel and the USA, respectively), the dataset at the Oncogenetics unit at the Sheba 
Medical center of male BRCA carriers, the dataset of a USA based study on male breast cancer cases, and the Italian National Study of Male Breast Cancer.

Israel. The study was approved by the Sheba Ethics Committee (SMC 1493-2000). A cross referencing of all individuals registered in the context of the Israeli National Cancer registry (INCR) since its establishment in 1960 with the International Classification of Disease (ICD 0-3) code C48.2, was carried out and the sex of the individuals was obtained. No other details were available from this source. The INCR, a passive, national, population-based cancer registry, was established in 1960. Since 1982, reporting on all cancer cases to the INCR is compulsory by law. The INCR completeness with respect to patients with solid tumors is over $96.8 \%$ (12).

BRCA carriers. A cross-referencing of all male carriers of BRCA1 or $B R C A 2$ mutation genotyped and identified at the Oncogenetics Unit, at the Sheba Medical Center since 1996 with the INCR was performed, as previously described (13), using the specific ICD 03 codes and the histological subtypes relevant to PPSC: 8260/3: papillary adenocarcinoma, non-otherwise specified (NOS); 8441/3: serous cystadenocarcinoma, NOS; 8460/3: papillary serous cystadenocarcinoma.

The SEER program database. The SEER database (www.seer. cancer.gov-Research Data released April 2016, based on the November 2015 submission) was queried for ICD 0-3 code 48.2. Subsequent analysis was restricted to histological codes 8260/3: papillary adenocarcinoma, NOS; 8441/3: serous cystadenocarcinoma, NOS; 8460/3: papillary serous cystadenocarcinoma.

Italy. Male breast cancer (MBC) cases (either population- or clinicbased series) were recruited from Italian Investigation Centers in different areas of the country in the framework of the collaborative Italian Multicenter Study on MBC (14). For each MBC case, information on personal and family history of cancer and $B R C A$ mutational status was collected by a geneticist and validated by the relevant sources, mainly local Cancer and Mortality Registries. BRCA1 and BRCA2 mutation analysis was performed in the context of genetic counselling programs at the local medical centers for all MBC cases and unaffected relatives. The Italian Multicenter study on MBC was approved by the local Ethical Committee (Sapienza University of Rome, Protocol 264/12). Written informed consent was obtained from all study participants.

United States. MBC cases: MBC cases were recruited from State Cancer Registries in Utah and the surrounding intermountain states (Colorado, Idaho and Wyoming), an on-line male support group and referrals from physicians and family members. The participants were enrolled under Institutional Review Board approval (IRB 09180) and all signed informed consent. Cases referred by family members were from breast cancer families and the remaining cases were unselected for age or family history of breast cancer as previously described (15). Cancer status was through the individual, relatives, or the Utah Cancer Registry for those residing in Utah.

Male members of BRCA1 and BRCA2 families: Male individuals of families carrying pathogenic mutations in BRCA1 and BRCA2 were identified. From linkage in the Utah Population Database, within these families, information on cancer status was extracted. Cancer status for the participants was through the individual, relatives, or the Utah Cancer Registry.

\section{Results}

Israel. The INCR encompasses $\sim 800000$ entries since 1960, $\sim 45 \%$ of which refer to men. There were 220 cases of code C48.2 in the entire cohort: 219 in women and one in a man (male:female ratio $=0.0045$ ). In all likelihood, this was the same case described by Shmueli et al. in 2001 (11). None of the 237 BRCA1/BRCA2 carriers genotyped at the Oncogenetics Unit developed cancer bearing the same code during a mean of 9.2 years of follow up.

SEER database. The total number of malignancies registered in SEER was $8,234,845(4,227,600$ in males). Search of individuals diagnosed with code 48.2 yielded 7,087 cases, including 6,041 females and 1,046 males. Search restricted to histology codes given above resulted in 2,673 cases, including 2,668 females and five males (male:female ratio $=0.0018$ ) For men, age at diagnosis ranged from 57 to 73 years.

Italy. A total of 624 men affected with breast cancer, including nine BRCAl and 79 BRCA2 mutation carriers were recruited. Age at first breast cancer diagnosis ranged between 22 and 91 years, with a mean age of 61.6 years. An additional 219 male BRCA mutation carriers who were family relatives of male breast cancer cases, including 107 with $B R C A 1$ and 112 with $B R C A 2$ mutation were recruited. Age at recruitment ranged between 20 and 86 years, with a mean age of 47 years. At the time of BRCA genotyping or diagnosis of breast cancer, none was diagnosed with PPSC. No follow-up data were available for these individuals.

United States. One hundred and fifteen male breast cancer cases were recruited from the above-mentioned sources (see Materials and Methods above): the 10 cases referred by family members were from breast cancer families and the remaining 105 cases were unselected for age or family history of breast cancer. Within the 105 male breast proband families, there were 1966 males. From 203 families carrying pathogenic mutations in BRCA1 and BRCA2, there were a total of 7817 males for whom we had information on cancer status.

Of a total of 9783 males in our BRCAl and BRCA2 families or families ascertained for a male-breast proband, there were no recorded or reported cases of PPSC at the time of last contact or cancer registry linkage in 2002. No followup data were available for these individuals.

\section{Discussion}

This study supports the common notion that PPSC is indeed a rare disease, with the overwhelming majority of diagnosed cases being women. It is also apparent that being a male $B R C A 1$ or a $B R C A 2$ mutation carrier or even being clinically defined as having high cancer risk with no known $B R C A$ 
mutation in men is not associated with any clinically significant risk for developing this rare entity.

Peritoneal epithelial cells originate from the embryonic mesonephric structures (16) in both men and women. Certain male reproductive organs differentiate from the mesonephric system, including the epididymis, vas deferens, and seminal vesicles. Yet PPSC is extremely rare in males.

The differences in the rates between men and women could be accounted for by several factors including: hormonal exposure, genetic factors and the presence of Mullerian-derived intraperitoneal cell implants that may be gender specific. Indeed, Dubeau (17) suggested that the cell of origin in ovarian carcinoma and PPSC is derived from the primary and secondary Mullerian system (paramesonephric) and not from the epithelial cells lining the ovaries and peritoneum. One plausible explanation to account for the vastly divergent rates in males and females of PPSC diagnosis is that this tumor indeed arises from cells implanted on the peritoneum that may originate, at least in part, from cells that were shed from the fallopian tubes in women, or from the secondary Mullerian system (microscopic structures lined by Mullerian epithelium). Male sertoli cells, secrete Mullerian-inhibiting substances (MIS) starting at the seventh week of gestation, inducing Mullerian system regression. Levels peak early in postnatal life, decline during puberty due to the inhibitory effect of testosterone, and remain relatively stable through adulthood and always higher than the levels in women (18). MIS may contribute to the lack of growth of Müllerian system-derived tumors in males. Indeed, monoclonal antibody anti-Müllerianinhibiting substance type II receptor (MISRII) showed promise in treating ovarian granulosa cells and epithelial cell tumors in mice (19). Vaccination with anti MISRII significantly inhibited tumor growth when administered either prophylactically or therapeutically to mice (20). Such a notion of the pathogenesis of PPSC is consistent with the diagnosis of PPSC within a few years following RRBSO.

Another plausible explanation to account for PPSC diagnosis in men is persistent remnant Mulllerian duct syndrome (PMDS MIM \# 261550). This is a rare genetic condition leading to the presence of Müllerian duct derivatives in phenotypical males. It is hypothesized that PMDS is the result of MIS deficiency, receptor mutation, or pure lack of expression of MIS in utero (21). Yet all these hypotheses should be viewed as speculative since there are no data presented herein to substantiate these notions. Furthermore, at least some PPSCs that have been analyzed histopathologically seem to have distinct molecular features compared with epithelial and intra-fallopian tumors (22).

The limitations of this study should be noted. For BRCA carriers from the USA and Italy, there were no follow-up data, so the lifetime risk for developing PPSC in these highrisk individuals is still unknown. The risk for developing ovarian cancer depends, in part, on the location of the specific mutation, which may be different in the various $B R C A$ mutation carriers from ethnically-diverse populations compared with the rather limited spectrum of mutations in Jewish Ashkenazi individuals. An additional limitation is that there was no independent pathology review of all the reported cases. Thus, in the reported Israeli male case, p53 immunohistochemistry, the most common pathological feature of PPSC (22) was not rechecked (11).

In conclusion, PPSC is a rare neoplasm, seemingly not associated with $B R C A$ mutations in men, and in women, the cell of origin may be of Müllerian tube origin, possibly with some contribution of fallopian tube-originated epithelial cell implants, especially in $B R C A$ mutation carriers.

\section{Conflicts of Interest}

All Authors declare that they have no conflicts of interest in regard to this study.

\section{Funding}

This research did not receive any specific grant from funding agencies in the public, commercial, or not-for-profit sectors.

\section{References}

1 Goodman MT and Shvetsov YB: Incidence of ovarian, peritoneal, and fallopian tube carcinomas in the United States, 1995-2004. Can Epidemiol Biom Prev 18(1): 132-139, 2009.

2 Ben-Baruch G and Sivan E, Moran O, Rizel S, Menczer J and Seidman DS: Primary peritoneal serous papillary carcinoma: a study of 25 cases and comparison with stage III-IV ovarian papillary serous carcinoma. Gynecol Oncol 160(3): 393-396, 1996.

3 Muto MG, Welch WR, Mok SC, Bandera CA, Fishbaugh PM, Tsao SW, Lau CC, Goodman HM, Knapp RC and Berkowitz RS: Evidence for a multifocal origin of papillary serous carcinoma of the peritoneum. Cancer Res 55(3): 490-492, 1995.

4 Seidman JD and Wang BG: Evaluation of normal-sized ovaries associated with primary peritoneal serous carcinoma for possible precursors of ovarian serous carcinoma. Gynecol Oncol 106: 201-206, 2007.

5 Tong GX, Chiriboga L, Hamele-Bena D and Borczuk AC: Expression of PAX2 in papillary serous carcinoma of the ovary: Immunohistochemical evidence of fallopian tube or secondary Mullerian system origin? Mod Pathol 20: 856-863, 2007.

6 Casey MJ, Synder C, Bewtra C, Narod SA, Watson P and Lynch HT: Intra-abdominal carcinomatosis after prophylactic oophorectomy in women of hereditary breast ovarian cancer syndrome kindreds associated with BRCA1 and BRCA2 mutations. Gynecol Oncol 97(2): 457-467, 2005.

7 Rebbeck TR, Lynch HT, Neuhausen SL, Narod SA, Van't Veer L, Garber JE, Evans G, Isaacs C, Daly MB, Matloff E, Olopade OI, Weber BL for Prevention and Observation of Surgical End Points Study Group: Prophylactic oophorectomy in carriers of BRCA1 or BRCA2 mutations. N Engl J Med 346: 1616-1622, 2002. 
8 Gotlieb WH, Barchana M, Ben-Baruch G and Friedman E: Malignancies following bilateral salpingo-oophorectomy (BSO). Eur J Surg Oncol 32(10): 1231-1234, 2006.

9 Canbay E, Ishibashi H, Sako S, Kitai T, Nishino E, Hirano M, Mizumoto A, Endo Y, Ogura S and Yonemura Y: Photodynamic detection and management of intraperitoneal spreading of primary peritoneal papillary serous carcinoma in a man: report of a case. Surg Today 44(2): 373-377, 2014.

10 Shah IA, Jayram L, Gani OS, Fox IS and Stanley TM: Papillary serous carcinoma of the peritoneum in a man: a case report. Cancer 82(5): 860-856, 1998.

11 Shmueli E, Leider-Trejo L, Schwartz I, Aderka D and Inbar M: Primary papillary serous carcinoma of the peritoneum in a man. Ann Oncol 12(4): 563-567, 2001.

12 Fishler Y, Keinan-Boker L and Ifrah A (eds.): The Israel National Cancer Registry - Completeness and Timeliness of the Data. Israel Center for Disease Control, Israel Ministry of Health. Publication \# 365, February 2017, Israel (in Hebrew).

13 Laitman Y, Keinan Boker L, Liphsitz I, Weissglas-Volkov D, Litz-Philipsborn S, Schayek H and Friedman E: Cancer risks in Jewish male $B R C A 1$ and $B R C A 2$ mutation carriers. Breast Cancer Res Treat 150(3): 631-635, 2015.

14 Silvestri V, Rizzolo P, Scarnò M, Chillemi G, Navazio AS, Valentini V, Zelli V, Zanna I, Saieva C, Masala G, Bianchi S, Manoukian S, Barile M, Pensotti V, Peterlongo P, Varesco L, Tommasi S, Russo A, Giannini G, Cortesi L, Viel A, Montagna M, Radice P, Palli D and Ottini L: Novel and known genetic variants for male breast cancer risk at 8q24.21,9p21.3, 11q13.3 and 14q24.1: results from a multicenter study in Italy. Eur $\mathbf{J}$ Cancer 51(16): 2289-2295, 2015.

15 Ding YC, Steele L, Kuan CJ, Greilac S and Neuhausen SL: Mutations in BRCA2 and PALB2 in male breast cancer cases from the United States. Breast Cancer Res Treat 126(3): 771-778, 2011.

16 Rodriguez M and Dubeau L: Ovarian tumor development: insights from ovarian embryogenesis. Eur J Gynaecol Oncol 22(3): 175-183, 2001.
17 Dubeau L: The cell of origin of ovarian epithelial tumours. Lancet Oncol 9(12): 1191-1197, 2008.

18 Lindhardt Johansen M, Hagen CP, Johannsen TH, Main KM, Picard JY, Jorgensen A, Rajpert-De Meyts E and Juul A: AntiMullerian hormone and its clinical use in pediatrics with special emphasis on disorders of sex development. Int $\mathbf{J}$ Endocrinol 2013: 198698, 2013

19 Kersual N, Garambois V, Chardes T, Pouget JP, Salhi I, BascoulMollevi C, Bibeau F, Busson M, Vié H, Clémenceau B, Behrens CK, Estupina P, Pèlegrin A and Navarro-Teulon I: The human Mullerian-inhibiting substance type II receptor as immunotherapy target for ovarian cancer. Validation using the mAb 12G4. mAbs 6(5): 1314-1326, 2014.

20 Sakalar C, Mazumder S, Johnson JM, Altuntas CZ, Jaini R, Aguilar R, Naga Prasad SV, Connolly DC, Tuohy VK: Regulation of murine ovarian epithelial carcinoma by vaccination against the cytoplasmic domain of anti-Mullerian hormone receptor II: J Immun Res 2015: 630287, 2015.

21 Pulido L, Iwasiuk G, Sparkuhl M, Bui D and Springs H: Persistent Mullerian duct syndrome presenting in an incarcerated recurrent inguinal hernia with hydrocele. Urol Case Rep 12: 47 48, 2017.

22 Kuhn E, Kurman RJ, Vang R, Sehdev AS, Han G, Soslow R, Wang TL and Shih L-M: TP53 mutations in serous tubal intraepithelial carcinoma and concurrent pelvic high-grade serous carcinoma-evidence supporting the clonal relationship of the two lesions. J Pathol 226(3): 421-426, 2012.

Received April 21, 2017

Revised May 2, 2017

Accepted May 9, 2017 